\title{
Conductas sexuales en jóvenes mexicanos durante el confinamiento por COVID-19
}

\author{
Marily Daniela Amaro-Hinojosa; Vicente Jiménez-Vázquez²; Liliana Leticia Juárez-Mediana³; \\ Reyna Torres-Obregon ${ }^{4}$; Claudia Orozco-Gómez ${ }^{5 *}$
}

\begin{abstract}
RESUMEN
Introducción: El confinamiento por la pandemia de coronavirus (COVID-19) originó cambios en la atención a la salud sexual y reproductiva, así como en las conductas sexuales de la población. Objetivo: Describir las conductas sexuales de los jóvenes mexicanos durante el confinamiento por COVID-19. Metodología: Diseño descriptivo transversal, se conformó la muestra por 613 jóvenes de 18 a 28 años de México, el muestreo fue no probabilístico por conveniencia, se realizó una encuesta en línea. Se aplicó una cédula de datos y un cuestionario de conductas sexuales adaptado para este estudio. El análisis de los datos se realizó en el paquete estadístico SPSS versión 25 , a través de estadística descriptiva e inferencial como fue la prueba t de Student. Resultados: Las principales conductas encontradas durante el confinamiento por coronavirus fueron: la masturbación $49.9 \%$, ver pornografía $43.2 \%$, sexting $33.9 \%$, cibersexo $17.9 \%$ y tener sexo oral, vaginal y/o anal en $46.8 \%$. Conclusiones: La conducta sexual de los jóvenes se modificó durante el confinamiento comparado con el último año. Estos hallazgos brindan evidencia para el desarrollo de intervenciones de Enfermería para la promoción de la salud sexual ante situaciones de crisis en salud como lo es el COVID-19.
\end{abstract}

Palabras clave: Conducta sexual; Infecciones por Coronavirus; Confinamiento involuntario; Adulto Joven (DeCS).

1. Doctora en Ciencias de Enfermería. Universidad Autónoma de Chihuahua, Facultad de Enfermería y Nutriología, https://orcid.org/0000-0002-3250-492X

2. Doctor en Ciencias de Enfermería. Universidad Autónoma de Chihuahua, Facultad de Enfermería y Nutriología, http://orcid.org/0000-0001-5661-494X

3. Doctora en Ciencias de Enfermería. Universidad Autónoma de Tamaulipas, Facultad de Enfermería Nuevo Laredo, https://orcid.org/0000-0002-9656-4198

4. Doctora en Ciencias de Enfermería. Universidad Autónoma de Coahuila, Facultad de Enfermería, Unidad Saltillo, https://orcid.org/0000-0003-3546-6970

5. Doctora en Ciencias de Enfermería. Universidad Autónoma de Chihuahua, Facultad de Enfermería y Nutriología, https://orcid.org/0000-0001-94326404

*Autor para correspondencia: clorozco@uach.mx

Recibido: $14 / 10 / 2020$

Aceptado: 03/11/2020 


\section{Sexual behaviors in Mexican youth during COVID-19 confinement}

Introduction: Confinement due to the coronavirus pandemic (COVID-19) caused changes in sexual and reproductive health care, as well as in the sexual behaviors of the population. Objective: Describe the sexual behaviors of young Mexicans during COVID-19 confinement. Methodology: Cross-sectional descriptive design, where the sample consisted of 613 Mexican young people ranging from 18 to 28 years of age; sampling was non-probabilistic by convenience, an online survey was conducted. A data questionnaire and a sexual behavior questionnaire adapted for this study were applied. Data analysis was performed with the SPSS version 25 statistical package, using descriptive and inferential statistics such as Student's t-test. Results: The main behaviors found during coronavirus confinement were the following: Masturbation 49.9\%, viewing pornography $43.2 \%$, sexting $33.9 \%$, cybersex $17.9 \%$ and oral, vaginal, and/or anal sex $46.8 \%$. Conclusions: Youth sexual behavior changed during confinement compared to the former year. These findings provide evidence for the implementation of nursing interventions for the promotion of sexual health during health crisis situations such as COVID-19.

Keywords: Sexual behavior; coronavirus infections; involuntary confinement; young adult (DeCS). 


\title{
Comportamentos sexuais em jovens mexicanos durante o confinamento COVID-19
}

\begin{abstract}
ABSTRATO
Introdução: O confinamento devido à pandemia do coronavírus (COVID-19) causou mudanças nos cuidados com a saúde sexual e reproductiva, bem como nos comportamentos sexuais da polulação. Objetivo: Descrever os comportamentos sexuais de jovens mexicanos durnate o confinamento COVID19. Metodologia: Delinamiento transversal descritivo, onde a amostra foi composta por 613 jovens mexicanos de 18 a 28 anos; a amostragem era não probabilística por conveniência, uma pesquisa online foi conduzida. Foi aplicado um questionário de dados e um questionário de comportamento sexual adaptado para este estudo. A análise dos dados foi realizada como o pacote estatístico SPSS versão 25, utilizando estatística descritiva e inferencial, como o teste t de Student. Resultados: Os principais comportamentos encontrados durante o confinamento do coronavírus foram: Masturbação $49,9 \%$, ver pornografia $43,2 \%$, sexting $33.9 \%$, cibersexo $17.9 \%$, e sexo oral, vaginal e/our anal $46.8 \%$. Conclusões: O comportamento sexual dos jovens mudou durante o confinamento em comparação com o ano anterior. Esses achados fornecem evidencias para a implementação de intervenções de enfermagem para a promoção da saúde sexual durante situações de crise de saúde, como o COVID-19.
\end{abstract}

Palavras-chave: Comportamento sexual; infecções por coronavírus; confinamiento involuntário; jovem adulto (DeCS). 


\section{INTRODUCCIÓN}

El coronavirus SARS-Cov-2 que causa la enfermedad de COVID-19 fue notificado por primera vez en Wuhan China el 31 de diciembre del 2019, fue hasta el 11 de marzo del 2020 que se declara la pandemia por Sars-Cov-2 ${ }^{(1,2)}$. Este suceso trajo consigo una serie de dificultades sociales, económicas y en la salud a las personas (3). Como parte de la mitigación de la pandemia las autoridades de salud a nivel mundial y en México establecieron el distanciamiento social, como medida para prevenir el COVID-19, el cual se consideró una de las estrategias más efectivas, puesto que es una enfermedad de fácil transmisión y propagación, el contacto cercano entre personas incluyendo relaciones sexuales y los encuentros cara a cara se restringieron, como consecuencia, la conducta sexual se modificó y adquirió nuevas formas de satisfacer el placer sexual ${ }^{(4-7)}$.

Diversos estudios han documentado la conducta sexual durante el confinamiento en población adulta, donde se observó la disminución de la frecuencia de las relaciones sexuales, pero se incrementó la masturbación ( 7 , ${ }^{8)}$. Además, se incorporan diferentes formas de satisfacer sus deseos sexuales como el cibersexo, es decir el uso de Internet con fines sexuales, como lo es la pornografía y sexting ${ }^{(7,}$

9). Cabe señalar que el cibersexo se asocia con conductas sexuales de riesgo en jóvenes como en el sexo oral, sexo con parejas ocasionales y relaciones sexuales bajo los efectos de alcohol $\mathrm{u}$ otras drogas ${ }^{(10)}$.

Durante la pandemia se encontró que quienes tenían relaciones sexuales bajo efecto de drogas ilegales tenían mayor número de parejas casuales por mes y una mayor probabilidad de tener sexo anal sin condón y quienes consumían alcohol tenían mayor actividad sexual ${ }^{(11,12)}$. Dichas conductas son de riesgo para adquirir infecciones de transmisión sexual, además de incrementar el riesgo de la transmisión del Sars-Cov-2 por el contacto oral/anal ${ }^{(13,14)}$.

Para disminuir el riesgo de contagio y promover el bienestar sexual de las personas durante la pandemia se establecieron recomendaciones entre las que destacan: a) practicar la masturbación, b) tener actividad sexual a través de plataformas digitales y c) mantener relaciones sexuales con parejas con las que ha permanecido durante el período del confinamiento por coronavirus ${ }^{(15)}$. Coca y cols. llevaron a cabo un estudio en jóvenes de entre 18 a 23 años, los cuales rompieron las normas de distanciamiento social para tener un encuentro sexual ${ }^{(16)}$.

Cabe mencionar, que la evidencia relacionada con la conducta sexual de los jóvenes durante la pandemia es escasa, por consiguiente se exhortó a través de medios de divulgación, documentar el impacto de aspectos relacionados con la salud sexual, un ejemplo de esto son las conductas sexuales (17) por lo que surge la pregunta de investigación ¿Cuáles son las conductas sexuales que los jóvenes mexicanos presentaron durante el confinamiento por COVID-19?

\section{Objetivo}

Describir las conductas sexuales que los jóvenes Mexicanos de 18 a 28 años durante el confinamiento por COVID-19.

\section{METODOLOGÍA}

\section{Diseño del estudio}

El diseño del estudio fue de tipo descriptivo transversal. La población fueron jóvenes de la República Mexicana, residentes en estados del norte del país (Chihuahua, Saltillo, Nuevo León y Tamaulipas) norte occidente (Baja California, Sinaloa y Sonora), centro norte (Jalisco, Aguascalientes, Guanajuato, San Luis Potosí, Querétaro y Zacatecas) centro (Puebla, México y Tlaxcala) y sur (Chiapas, Campeche, Tabasco, Veracruz, Oaxaca y Yucatán), se incluyeron 613 participantes. La muestra se calculó para un intervalo de confianza del $99 \%$ y un margen de error del $5.3 \%$. Sólo se incluyeron aquellos jóvenes que se encontraban en el rango edad de 18 a 28 años. El muestreo fue no probabilístico por conveniencia. Como criterios de eliminación se consideraron: 1) no dar su consentimiento de participación en el estudio y 
2) participantes que dejaron los instrumentos con respuestas incompletas.

\section{Instrumentos}

Las mediciones consistieron en la aplicación de un cuestionario en línea, el cual se conformó por dos apartados: 1) Cédula de datos personales en la que se recaudó información como el estado de procedencia, género, edad, estado civil, escolaridad y ocupación. 2) Cuestionario de Conductas Sexuales de los jóvenes adaptado para fines de este estudio, mismo que se basó en la Escala de Conductas Sexuales de Riesgo, validado en idioma español en jóvenes adolescentes (18), el cuestionario se conformó por 9 ítems puntuados con una escala tipo dicotómica. Algunos ejemplos de reactivos son "¿Has practicado sexting, durante el periodo de contingencia por COVID-19?; ¿Has tenido relaciones sexuales orales, vaginales y/o anales durante el periodo de contingencia por COVID19?; Desde que inicio el periodo de contingencia COVID-19 ¿Cuántas veces tú o tu pareja usaron condón?; Desde el inicio del periodo de contingencia COVID-19 ¿De qué lugar obtuviste los condones?, etc.).

\section{Ética del estudio}

El estudio estuvo sujeto a las normas dispuestas en la Ley General de Salud en materia de investigación en seres humanos (19) y fue aprobado por el Comité de Ética e Investigación de la Facultad de Enfermería y Nutriología de la UACH: Folio SIP-CI/2020/04.

\section{Procedimiento}

Al obtener los permisos correspondientes, se inició con la recolección de los datos en línea. El consentimiento informado y los instrumentos fueron capturados y estuvieron disponibles en la plataforma Google Formularios, se difundió la liga que permitió el acceso a éstos en la red social Facebook del 22 de junio al 08 de julio del 2020, en este período de tiempo fue compartido por esta red, hasta lograr recolectar la muestra, se obtuvieron 625 encuestas, de las cuales solo se incluyeron en el análisis 613 , ya que se eliminaron 12 encuestas por no dar su consentimiento para participar en el estudio y presentar respuestas incompletas.

En el consentimiento informado, se incluyó información del estudio como objetivo, protección del anonimato del participante, así como la confidencialidad de los datos y los posibles riesgos de participar en la investigación, finalmente se colocó el contacto del investigador, si el participante estaba de acuerdo, seleccionaba la opción "acepto", acto seguido accedía a la cédula de datos sociodemográficos y al cuestionario sobre conductas sexuales, al finalizar, el participante tenía que elegir la opción de "enviar" el cuestionario para que sus datos estuvieran disponibles para los investigadores. Cabe mencionar que no se solicitó ningún dato de identificación personal, ni información que evitaran garantizar su anonimato.

\section{Análisis estadístico}

El análisis de los datos se realizó en el paquete estadístico SPSS versión 25. Para conocer las características sociodemográficas de los participantes, en la descripción de las variables categóricas del estudio se utilizaron frecuencias y porcentajes. Se emplearon medidas de tendencia central y dispersión en las variables numéricas. Se realizó como análisis adicional la prueba $t$ Student para pruebas independientes que permitió observar las diferencias existentes entre hombres y mujeres sexualmente activos durante el confinamiento por COVID-19.

\section{RESULTADOS}

Participaron 613 jóvenes de diferentes regiones del país, en su mayoría eran originarios del norte de México (53.5\%); seguido de la región sur (23\%); la media de edad de los participantes fue 22 años $(D E=2.85)$. Se observó que $75.2 \%$ pertenecían al sexo femenino y $54.8 \%$ eran estudiantes, 90.9\%; se percibían en un nivel socioeconómico medio, el estado civil predominante fue soltero con $82.9 \%$ y $85.3 \%$ mencionaron no tener hijos. En la Tabla 1, se 
presentan los datos sociodemográficos de los participantes.

Tabla 1. Características sociodemográficas de los jóvenes mexicanos

\begin{tabular}{|c|c|c|}
\hline Variable & $f$ & $\%$ \\
\hline \multicolumn{3}{|l|}{ Región del país de origen } \\
\hline Norte & 328 & 53.5 \\
\hline Norte occidente & 98 & 16.0 \\
\hline Centro & 20 & 3.3 \\
\hline Centro norte & 26 & 4.2 \\
\hline Sur & 141 & 23.0 \\
\hline \multicolumn{3}{|l|}{ Sexo del participante } \\
\hline Masculino & 152 & 24.8 \\
\hline Femenino & 461 & 75.2 \\
\hline \multicolumn{3}{|l|}{ Ocupación } \\
\hline Trabaja & 119 & 19.4 \\
\hline Estudia & 336 & 54.8 \\
\hline Trabaja y estudia & 126 & 20.6 \\
\hline Ni trabaja, ni estudia & 32 & 5.2 \\
\hline \multicolumn{3}{|c|}{ Nivel socioeconómico percibido } \\
\hline Alto & 7 & 1.1 \\
\hline Medio & 557 & 90.9 \\
\hline Bajo & 49 & 8.0 \\
\hline \multicolumn{3}{|l|}{ Estado civil } \\
\hline Soltero & 508 & 82.9 \\
\hline Casado & 53 & 8.6 \\
\hline Unión libre & 48 & 7.8 \\
\hline
\end{tabular}


Hijos

Si

No
90

523
14.7

85.3

Orientación sexual

Heterosexual

534

87.1

32

Bisexual

47

Pareja actualmente

Si

No
379

234
61.8

38.2

Fuente: cédula de datos, $f=$ frecuencia, $\%=$ porcentaje, $n=613$

En la Tabla 2, se muestran las frecuencias y porcentajes de las conductas sexuales que los jóvenes llevaron a cabo alguna vez en la vida, en el último año y durante la contingencia por COVID-19, entre las que se destacan: la masturbación (49.9\%), tener sexo oral, vaginal y/o anal (46.8\%), ver pornografía (43.2\%), sexting (33.9\%) y cibersexo (17.9\%).

Tabla 2. Conductas sexuales en los jóvenes mexicanos durante el confinamiento por COVID-19

\begin{tabular}{|c|c|c|c|c|}
\hline \multirow[t]{2}{*}{ Variable } & \multicolumn{2}{|c|}{ Si } & \multicolumn{2}{|c|}{ no } \\
\hline & $f$ & $\%$ & $f$ & $\%$ \\
\hline \multicolumn{5}{|l|}{ Masturbación } \\
\hline Alguna vez en la vida & 440 & 71.8 & 173 & 28.2 \\
\hline En el último año & 400 & 65.3 & 213 & 34.7 \\
\hline Durante el confinamiento COVID-19 & 306 & 49.9 & 307 & 50.1 \\
\hline \multicolumn{5}{|l|}{ Ver pornografía } \\
\hline Alguna vez en la vida & 449 & 73.2 & 164 & 26.8 \\
\hline En el último año & 338 & 55.1 & 275 & 44.9 \\
\hline
\end{tabular}


Sexting

Alguna vez en la vida

En el último año

Durante el confinamiento COVID-19

Cibersexo

Durante el confinamiento COVID-19
346

268

208

33.9

405

66.1

43.6

56.3

$43.7 \quad 345$

Sexo

Alguna vez en la vida

Durante el confinamiento COVID-19
511

287
83.4

102

46.8

Fuente: Cuestionario de conductas sexuales, $f=$ frecuencia, $\%=$ porcentaje, $n=613$

De los 287 jóvenes que tuvieron relaciones sexuales durante el confinamiento por COVID-19, solo 283 manifestaron algunas de las conductas sexuales. En la Tabla 3 se observa el uso de condón en jóvenes que han tenido sexo durante el confinamiento por COVID-19, en promedio lo utilizaron 4.16 veces $(D E=8.26)$.

Tabla 3. Conductas sexuales de los jóvenes que han tenido sexo durante el confinamiento por COVID-19

\begin{tabular}{lccccccc}
\hline Variable & $n$ & $\bar{X}$ & Mediana & Moda & DE & Val. mín. & Val. máx. \\
\hline $\begin{array}{l}\text { Número de } \\
\text { personas con las } \\
\text { que has tenido }\end{array}$ & 283 & 1.41 & 1 & 1 & 4.12 & 1 & 70 \\
sexo & & 10.86 & 5 & 1 & 14.29 & 1 & 100 \\
$\begin{array}{l}\text { Número de veces } \\
\text { que has tenido } \\
\text { sexo }\end{array}$ & 283 & & 1 & 0 & 8.26 & 0 & 59 \\
$\begin{array}{l}\text { Número de veces } \\
\text { que has usado } \\
\text { condón }\end{array}$ & 283 & 4.16 & & & & & \\
\hline
\end{tabular}

Fuente: Cuestionario de conductas sexuales, $\bar{X}=$ media, $D E=$ desviación estándar, Val. $m i n .=$ valor mínimo, Val. máx. $=$ valor máximo; $n=283$ 
Posteriormente al comparar las diferencias entre hombres y mujeres respecto al número de personas con las que han tenido sexo $(t(285)=-.09, p=0.97)$, número de veces que han tenido sexo $(t(67.5)=1.56, p=0.12) \mathrm{y}$ número de veces que has usado condón ( $t$ (67.1) $=1.05, p=0.29)$, no se encontraron diferencias significativas.

Se encontró que $87.6 \%$ (248) de los jóvenes mencionaron tener relaciones sexuales con una pareja estable, $8.8 \%$ (25) ocasional, y $3.5 \%$ (10) ambas. En la Tabla 4, se describen frecuencias y porcentajes de los métodos anticonceptivos que utilizaron los jóvenes que mantuvieron relaciones sexuales durante el confinamiento por COVID-19; 84.5\% nunca tuvieron dificultad para acceder a métodos anticonceptivos, en contraste con $2.5 \%$ que siempre tuvo dificultad.

Tabla 4. Métodos anticonceptivos utilizados por los jóvenes que han tenido sexo durante el confinamiento por COVID-19

\begin{tabular}{|c|c|c|}
\hline Variable & $f$ & $\%$ \\
\hline \multicolumn{3}{|c|}{ Dificultad para acceder a métodos anticonceptivos } \\
\hline Siempre & 7 & 2.5 \\
\hline Casi siempre & 5 & 1.8 \\
\hline Algunas veces & 32 & 11.3 \\
\hline Nunca & 239 & 84.5 \\
\hline \multicolumn{3}{|l|}{ Lugar donde obtuviste condones } \\
\hline Centro de salud-hospital & 25 & 8.8 \\
\hline Farmacia-consultorio & 90 & 31.8 \\
\hline Supermercados & 38 & 13.4 \\
\hline Tiendas en línea & 1 & .4 \\
\hline Mi casa & 25 & 8.8 \\
\hline No utilice condón & 104 & 36.7 \\
\hline \multicolumn{3}{|c|}{ Uso de métodos anticonceptivos, excluyendo condón } \\
\hline Métodos naturales & 73 & 25.8 \\
\hline Métodos químicos-hormonales & 53 & 18.7 \\
\hline DIU & 18 & 6.4 \\
\hline Métodos definitivos & 1 & 0.4 \\
\hline
\end{tabular}


El lugar del que obtuvieron los preservativos en este período de tiempo fue una farmacia o consultorio (31.8\%). Sin embargo, $36.7 \%$ refirieron no utilizar condón cuando tenían relaciones sexuales, adicionalmente 48.8\% mencionaron no utilizar métodos anticonceptivos diferentes al condón. El método anticonceptivo más utilizado, excluyendo al condón fueron los métodos naturales (25.8\%).

\section{DISCUSIÓN}

La presente investigación mostró la conducta sexual que tuvieron jóvenes de diversos estados del país (en su mayoría del norte y norteoccidente) durante la contingencia a causa del COVID-19. En cuanto a las características sociodemográficas la mayoría de los participantes eran estudiantes de educación superior del sexo femenino, solteros, sin hijos y con pareja. Un estudio similar abordó el comportamiento sexual en jóvenes durante la pandemia en China, con diferencia de que la mayoría de los participantes fueron del sexo masculino (20).

De acuerdo con el objetivo general, que fue describir la conducta sexual de los jóvenes mexicanos de 18 a 28 años durante el confinamiento por COVID-19, los resultados señalan disminución en la frecuencia con la que practicaban las conductas sexuales, específicamente en la masturbación, el consumo de pornografía y el sexting. Los hallazgos son interesantes dado a que por el confinamiento y las restricciones sociales se esperaría que estas prácticas aumentaran al no ser de contacto directo. Se ha documentado que cuando disminuyen las oportunidades para tener sexo directo, las personas suelen recurrir al internet con el fin de realizar actividades individuales y en línea para satisfacer sus necesidades sexuales como puede ser a través de la masturbación (21).
Respecto al consumo de pornografía un estudio reportó un incremento de esta conducta durante el punto culminante del confinamiento, dicho aspecto fue atribuido a la presencia de ansiedad y estados de ánimo negativos en el individuo ocasionado por el confinamiento, esto generó un consumo descontrolado de pornografía, lo cual se considera como una conducta de adaptación sexualizada (22). No obstante, dicho hallazgo no concuerda con los resultados de este estudio.

Con relación al sexting, dado a que se considera una práctica creciente y de mayor frecuencia en la población joven ${ }^{(23)}$, se esperaría un aumento durante el confinamiento por el COVID-19, sin embargo, en este estudio se encontró una disminución de esta práctica. En este sentido, existe evidencia que el sexting representó una estrategia de afrontamiento temporal al inicio de la pandemia ${ }^{(7)}$. Por otra parte, los jóvenes no heterosexuales parecen estar más involucrados en el envío y recepción de contenido sexual, que aquellos que no se identifican como heterosexuales (24). Cabe mencionar que en este estudio la mayor parte de la muestra se identificó como heterosexual.

La disminución de las prácticas sexuales antes mencionadas en la población estudiada, probablemente está relacionado a la falta de privacidad en el confinamiento o bien por la reducción de la libido, lo cual se reportó en otras investigaciones (20). En este estudio se identificó disminución de la frecuencia de las relaciones sexuales, este hallazgo coincide con lo reportado con un estudio realizado en China, donde los participantes refirieron una disminución en el número de parejas sexuales y en la frecuencia con que tuvieron relaciones sexuales ${ }^{(25)}$. Así mismo, la Asociación Mexicana para la Salud Sexual reportó una disminución en la frecuencia de las relaciones sexuales ${ }^{(9)}$. Los resultados de nuestra investigación podrían deberse a la disminución en la privacidad percibida, hay que hacer notar que en México, se considera que la media de edad para independizarse es cerca de los 30 años, cabe 
recordar que el promedio de edad de la muestra de este estudio fue de 22 años, más del $50 \%$ son estudiantes y la mayor parte son solteros, esta información podría indicar que los participantes del estudio viven con su familia, aspecto que podría reforzar el planteamiento anterior, es decir, la falta de un espacio físico propio y de privacidad, podría haber incidido en el decremento de prácticas sexuales durante la contingencia.

Otro aspecto que podría explicar la disminución de las prácticas sexuales en este estudio podría ser la presencia de estrés y ansiedad derivados del aislamiento prolongado. El estrés es uno de los principales responsables de la disminución del deseo sexual, tanto en hombres como en mujeres al disminuir los niveles de testosterona y aumento en los niveles de cortisol. En un estudio realizado durante la pandemia por COVID-19 los participantes reportaron más problemas de disminución de deseo sexual, problemas para excitarse y mantener una erección, en comparación con el periodo anterior al inicio de la pandemia (9).

Otro resultado corresponde al uso inconsistente del condón, esto podría estar relacionado a que la mayor parte de la muestra estudiada reportó tener pareja estable y por tanto, influir en la percepción de seguridad para aceptar tener sexo sin protección. Sin embargo, dicho hallazgo es relevante considerando que en México el $\mathrm{VIH} / \mathrm{Sida}$ se adquiere principalmente entre los 15 y 29 años y del total de los casos registrados $30.7 \%$ corresponde a la población joven, siendo la vía sexual el principal medio de transmisión en $96.3 \%$ de los casos ${ }^{(26)}$.

Adicionalmente, el contagio del COVID-19 a través del sexo vaginal o anal aún se desconoce, ahora bien, se sabe que otros coronavirus no se propagan fácilmente por estas vías. De acuerdo con las recomendaciones para la práctica sexual segura es importante evitar los besos y el sexo oral sin protección con personas que no sean cercanas o que vivan en otra casa (27).

Sobre el uso de métodos anticonceptivos, excluyendo el uso del condón, sólo el $25.1 \%$ de los jóvenes utilizó métodos químicos hormonales o DIU. Estos datos son similares a lo que reporta en un estudio en el que se señaló que las formas de control de la natalidad disminuyeron significativamente durante la pandemia entre los participantes (28). Otro dato identificado es que más de la cuarta parte de los jóvenes utiliza medios naturales, lo cual es preocupante debido a que no se conocen cifras de eficacia de este tipo de métodos, lo que podría conllevar a riesgo de embarazos no planificados y no deseados (29).

Las limitaciones de este estudio que se pueden identificar son que no fue posible identificar otros factores que podrían haber influido en el cambio de la conducta sexual durante el confinamiento debido a que fue de tipo descriptivo, el instrumento utilizado se diseñó con fines del estudio y no se determinó su validez y confiabilidad.

Se recomienda continuar estudios con poblaciones sometidas a aislamiento como los hombres privados de la libertad; realizar más estudios de seguimiento sobre nuevos datos respecto a la conducta sexual después de la pandemia. También, es importante idear intervenciones para atenuar las consecuencias nocivas del aislamiento derivadas por la pandemia por COVID-19 y fomentar las prácticas sexuales seguras.

\section{CONCLUSIONES}

Los resultados de este estudio permitieron identificar que se modificó la conducta sexual de jóvenes mexicanos de 18 a 28 años durante el confinamiento por COVID-19, específicamente se observó una disminución en las frecuencias de las conductas sexuales como: masturbación, sexting, ver pornografía y relaciones sexuales durante el confinamiento por COVID-19 en los jóvenes comparado con el último año. A pesar de ser un estudio descriptivo, los hallazgos del estudio podrían ser útiles para comprender la repercusión de la pandemia de COVID-19, el distanciamiento social físico y la conducta sexual en jóvenes. Sin embargo, el cambio no es uniforme y los resultados no son concluyentes, comparados 
con otras investigaciones, por lo que se sugiere continuar el estudio de estas variables.

\section{CONFLICTO DE INTERESES}

Los autores declaran que no existen conflictos de intereses en este manuscrito

\section{FINANCIAMIENTO}

Los autores declaran que no se recibió algún tipo de financiamiento para el desarrollo de esta investigación y manuscrito.

\section{REFERENCIAS BIBLIOGRÁFICAS}

1. Organización Mundial de la Salud. Brote por enfermedad de coronavirus (Covid-19) [Internet]. 2020 [consultado Octubre 2020]. Disponible en: https://www.who.int/es/emergencies/diseases/ novel-coronavirus-2019

2. Organización Mundial de la Salud. COVID-19: cronología de la actuación de la OMS. [Internet]. 2020 [consultado Octubre 2020]. Disponible en: https://www.who.int/es/news-room/detail/2704-2020-who-timeline---covid-19

3. Ibarra FP, Mehrad M, Di Mauro MG, Peraza MF, García CE, Nilforoushzadeh MA, Russo GI. Impact of the COVID-19 pandemic on the sexual behavior of the population. The vision of the east and the west. Int Braz J Urol. [Internet]. 2020 [consultado Octubre 2020]; 46(suppl.1):104$112 . \quad$ Disponible en: https://doi.org/10.1590/s1677-

5538.ibju.2020.s116

4. Ministerio de Salud Argentina. Recomendaciones para sexo seguro. [Internet]. 2020 [consultado Octubre 2020]. Disponible en: http://www.msal.gob.ar/images/stories/ryc/gra ficos/0000001468cnt-covid-

19_recomendaciones-para-sexo-seguro.pdf
5. Acuerdo por el que se establecen las medidas preventivas que se deberán implementar para la mitigación y control de los riesgos para la salud que implica la enfermedad por el virus SARSCoV2 (COVID-19). DOF Secretaría de Gobernación. [Internet]. 2020 [consultado Octubre 2020]. Disponible en: https://www.dof.gob.mx/nota_detalle.php?codi go $=5590339 \&$ fecha $=24 / 03 / 2020$

6. Qian $M$, Jiang J. COVID-19 and social distancing. J. Public Health [Internet]. 2020 [consultado Octubre 2020];1-3. Disponible en: https://doi.org/10.1007/s10389-020-01321-z

7. Lehmiller JJ. Garcia JR. Gesselman AN. Mark $\mathrm{KP}$. Less sex, but more sexual diversity: changes in sexual behavior during the COVID-19 coronavirus pandemic. Leisure Sciences [Internet]. 2020 [consultado Octubre 2020]. Disponible en: https://doi.org/10.1080/01490400.2020.177401 6

8. Yuksel B, Ozgor F. Effect of the COVID-19 pandemic on female sexual behavior. Int $J$ Gynaecol Obstet. [Internet]. 2020 [consultado Octubre 2020]; 150(1):98-102. Disponible en: https://obgyn.onlinelibrary.wiley.com/doi/epdf /10.1002/ijgo.13193

9. Asociación Mexicana para la Salud Sexual, AC. Sexualidad y COVI-19 Resultados de la Encuesta Amssac. (2020). [Internet]. 2020 [consultado Octubre 2020]. Disponible en: https://www.amssac.org/sexualidad-y-covid19-resultados-de-la-encuesta-amssac/

10. García-Barba M, Nebot-García JN, Juliá BG, Giménez-García C. Conductas sexuales de riesgo y uso del cibersexo. Comparación entre diferentes perfiles de uso del cibersexo. Ágora de Salut, [Internet]. 2018[consultado Octubre 2020]; 137-146. Disponible en: http://dx.doi.org/10.6035/AgoraSalut.2019.6.15

11. Starks TJ, Jones SS, Sauermilch D, Benedict $M$, Adebayo T, Cain D, Simpson KN. Evaluating the impact of COVID-19: A cohort comparison study of drug use and risky sexual behavior 
among sexual minority men in the U.S.A. Drug Alcohol Depend. [Internet]. 2020 [consultado Octubre 2020]; 8;216:108260. Disponible en: http://dx.doi.org/10.1016/j.drugalcdep.2020.10 8260

12. Jacob $L$, Smith $L$, Butler $L$, Barnett $Y$, Grabovac I, McDermott D, Armstrong N, Yakkundi A, Tully MA. Challenges in the Practice of Sexual Medicine in the Time of COVID-19 in the United Kingdom. J Sex Med. [Internet]. 2020 [consultado Octubre 2020]; 17(7):1229-1236. Disponible en: http://dx.doi.org/10.1016/j.jsxm.2020.05.001

13.Glynn TR, Operario D, Montgomery $M$, Almonte A, Chan PA. The Duality of Oral Sex for Men Who Have Sex with Men: An Examination Into the Increase of Sexually Transmitted Infections Amid the Age of HIV Prevention. AIDS Patient Care STDS. [Internet]. 2017 [consultado Octubre 2020];31(6):261-267. Disponible en http://dx.doi.org/10.1089/apc.2017.0027

14. Cipriano M, Giacalone A, Ruberti E. Sexual Behaviors During COVID-19: The Potential Risk of Transmission. Arch Sex Behav. [Internet]. 2020 [consultado Octubre 2020]; 49(5):14311432.Disponible en: http://dx.doi.org/10.1007/s10508-020-01757-0.

15. Turban JL, Keuroghlian AS, Mayer KH. Sexual Health in the SARS-CoV-2 Era. Ann Intern Med. [Internet]. 2020 [consultado Octubre 2020]; 1;173(5):387-389. Disponible en: http://dx.doi.org/10.7326/M20-2004

16. Coca RP, Gutiérrez HG, Limpias RF, Munckel GDI, Salvatierra ORM, Gonzalo FD. Confinamiento, distanciamiento social y vida sexual en tiempos de COVID-19. [Internet]. $2020 . \quad$ Disponible en: https://www.researchgate.net/publication/3440 26814_Confinamiento_distanciamiento_social_y _vida_sexual_en_tiempos_de_COVID-19

17. Scott-Sheldon LAJ, Mark KP, Balzarini RN, Welling LLM. Call for Proposals: Special Issue of Archives of Sexual Behavior on the Impact of COVID-19 on Sexual Health and Behavior. Arch
Sex Behav. [Internet] 2020 [consultado Octubre 2020];49(5):1393-1394. Disponible en: https://doi.org.mx/10.1007/s10508-020-017258.

18.Moral RJ. Validación local de una escala de conductas sexuales de riesgo en adolescentes escolarizados mexicanos. Revista Internacional de Psicología [Internet]. 2016 [consultado Mayo 2020]; 15(2):1-56. Disponible en: https://www.revistapsicologia.org/index.php/re vista/article/view/226/84

19. Ley General de Salud en Materia de Investigación para la Salud. [Internet]. México: Secretaría de Salud; 2014 [consultado 2020 Octubre]. Disponible en: http://www.salud.gob.mx/unidades/cdi/nom/c ompi/rlgsmis.html

20. 1. Li G, Tang D, Song B, Wang C, Qunshan S, Xu C, Geng H, Wu H, He X, Cao Y. Impact of the COVID-19 Pandemic on Partner Relationships and Sexual and Reproductive Health: CrossSectional, Online Survey Study. J Med Internet Res. [Internet] 2020. [consultado octubre 2020]; 22(8). Disponible en: https://www.jmir.org/2020/8/e20961/

21. Nixon PG, Düsterhöft I K. Sex in the digital age. New York: Routledge; 2018.

22. Jung $S$, Kneer J, Krueger T. The German COVID-19 Survey on Mental Health: Primary Results. MedRxiv. [Internet] 2020. [consultado octubre 2020]; https://doi.org/10.1101/2020.05.06.20090340

23. Ojeda $M$, Del-Rey $R$, Walrave $M$, Vandebosch, $H$. Sexting en adolescentes: Prevalencia y comportamientos. Comunicar: Revista Científica de Comunicación y Educación [Internet] 2020. [consultado octubre 2020]; 28(64): $\quad 9-19$. https://www.revistacomunicar.com/index.php?c ontenido $=$ detalles \&numero $=64 \&$ articulo $=64$ 2020-01

24. Van-Ouytsel, J, Walrave M, Ponnet, K. An exploratory study of sexting behaviors among 
heterosexual and sexual minority early adolescents. Journal of Adolescent Health, [Internet] 2019. [consultado octubre 2020]; 65(5): 621-626. Disponible en: https://doi.org/10.1016/j.jadohealth.2019.06.00 3

25. Li W, Li G, Xin C, Wang Y, Yang S. Challenges in the Practice of Sexual Medicine in the Time of COVID-19 in China. The journal of sexual medicine, [Internet] 2020. [consultado octubre 2020]; 17(7): 1225-1228. Disponible en: https://doi.org/10.1016/j.jsxm.2020.04.380

26. Centro Nacional para la Prevención y Control del VIH y el sida. Vigilancia Epidemiológica de casos de VIH/SIDA en México. Registro Nacional de Casos de SIDA. [Internet]2019. [consultado octubre 2020]. Disponible en: https://www.gob.mx/cms/uploads/attachment/ file/513720/RN_D_a_Mundial_sida_2019.pdf

27. Alves da Silva Lara L, Lima de Oliveira Marino FF, Abdo CH, Brendler J, Glina S. Safe sexual practices in the COVID-19 pandemic period. Sex Med. [Internet] 2020. [consultado Octubre 2020]. Disponible en: https://www.ncbi.nlm.nih.gov/pmc/articles/PM C7467058/

28. Yuksel B, Ozgor F. Effect of the COVID-19 pandemic on female sexual behavior. International Journal of Gynecology \& Obstetrics [Internet] 2020. [consultado octubre 2020]; 150(1):98-102. https://obgyn.onlinelibrary.wiley.com/doi/epdf /10.1002/ijgo.13193

29. Organización Mundial de la Salud. Planificación familiar. [Internet] 2020 [consultado Octubre 2020]. Disponible en: https://www.who.int/es/news-room/fact-

sheets/detail/family-planning-contraception
Copyright (C) 2020 SANUS

Artículo de acceso abierto distribuido bajo Licencia Creative Commons

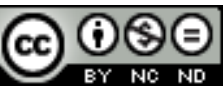

